\title{
Dermoscopy of Inflammatory Dermatoses (Inflammoscopy): An Up-to-Date Overview
}

\author{
Enzo Errichetti ${ }^{1}$
}

1 Institute of Dermatology, Santa Maria della Misericordia University Hospital, Udine, Italy

Key words: dermoscopy, differential diagnosis, general dermatology, inflammoscopy

Citation: Errichetti E. Dermoscopy of inflammatory dermatoses (inflammoscopy): an up-to-date overview. Dermatol Pract Concept. 2019;9(3):169-180. DOI: https://doi.org/10.5826/dpc.0903a01

Accepted: June 2, 2019; Published: July 31, 2019

Copyright: $\odot 2019$ Errichetti. This is an open-access article distributed under the terms of the Creative Commons Attribution License, which permits unrestricted use, distribution, and reproduction in any medium, provided the original author and source are credited.

Funding: None.

Competing interests: The author has no conflicts of interest to disclose.

Authorship: The author takes responsibility for this publication.

Corresponding author: Enzo Errichetti, MD, MSc, Institute of Dermatology, Santa Maria della Misericordia University Hospital, Piazzale Santa Maria della Misericordia, 15, 33100 Udine, Italy. Email: enzoerri@yahoo.it

ABSTRACT In addition to its use in pigmented and nonpigmented skin tumors, dermoscopy is gaining appreciation in assisting the diagnosis of nonneoplastic diseases, especially inflammatory dermatoses (inflammoscopy). In this field, dermoscopic examination should be considered as the second step of a "2-step procedure," always preceded by the establishment of a differential diagnosis on the basis of clinical examination. In this paper, we sought to provide an up-to-date overview on the use of dermoscopy in common inflammatory dermatoses based on the available literature data. For practical purposes, the analyzed dermatoses are grouped according to the clinical presentation pattern, in line with the 2-step procedure principle: erythematous-desquamative and papulosquamous dermatoses, papulokeratotic dermatoses, erythematous facial dermatoses, sclero-atrophic dermatoses, and miscellaneous.

\section{Introduction}

Dermoscopy is a fascinating bridge between clinical and histological examination that has become a key tool for the evaluation of pigmented and nonpigmented skin tumors because of its ability to reveal findings not visible to the naked eye [1,2]. Besides this classic application, it is gaining appreciation in areas other than dermato-oncology, especially inflammatory dermatology (inflammoscopy) [1,2].

While a well-established and structured approach for the analysis of dermoscopic images is available in the field of tumoral diseases, criteria and terminology used for inflammatory dermatoses in the literature are often variable, metaphoric, and poorly comprehensible, with consequent lack of a systematic analytic approach [1,2]. For this reason, a set of 5 dermoscopic parameters (with a total of 31 subitems) has been proposed by a consensus document of the International Dermoscopy Society as a basic guide to use in general dermatology [3]: (I) vessels (including morphology and distribution); (II) scales (including color and distribution); (III) follicular findings; (IV) "other structures" (structures other than vessels/scales; including color and morphology); and (V) "specific clues" (features that, when present, are strongly suggestive of only 1 diagnosis due to a strict dermoscopicpathological correlation).

As vascular structures and scales are the main characterizing dermoscopic features of inflammatory diseases, the selection of proper equipment is of utmost importance [1]. In this 


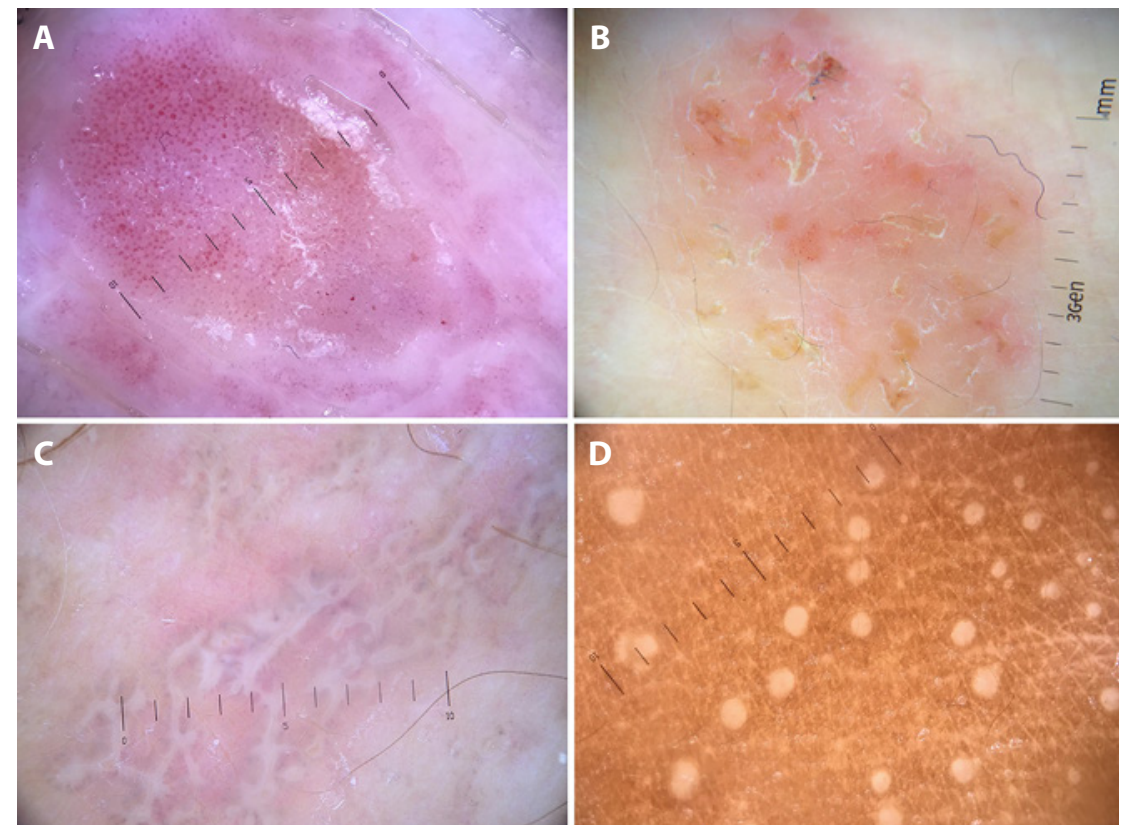

Figure 1. Uniform dotted vessels and diffuse white scales in psoriasis (A). Dotted vessels distributed in clusters and yellow scales and serocrusts in eczematous dermatitis (B). The typical Wickham striae of lichen planus; brownish dots are also visible around Wickham striae $(C)$. Lichen nitidus typically reveals roundish, well-defined, white areas devoid of physiological skin markings (D). [Copyright: @2019 Errichetti.]

regard, the use of noncontact polarized dermatoscopes is usually recommended as they preserve such findings; an interface fluid (eg, oil or gel) is sometimes required to enhance the visualization of structures covered by overlying scaling $[1,2]$.

Of note, dermoscopic assessment of inflammatory diseases should be considered as the second step of a " 2 -step procedure," always preceded by the establishment of a differential diagnosis on the basis of clinical examination $[1,2]$. Indeed, such conditions often display only poorly specific dermoscopic findings that, however, may be useful if used in the context of a specific differential diagnosis [1,2].

This paper aims to provide an up-todate overview on the use of dermoscopy in common inflammatory dermatoses based on the available literature data. For practical purposes, the analyzed dermatoses are grouped according to the clinical presentation pattern, in line with the 2-step procedure principle (ie, clinical differential diagnosis followed by dermoscopic analysis): erythematousdesquamative and papulosquamous dermatoses, papulokeratotic dermatoses, erythematous facial dermatoses, scleroatrophic dermatoses, and miscellaneous.

\section{Erythematous- Desquamative and Papulosquamous Dermatoses}

\section{Psoriasis}

Psoriasis is surely one of the dermatoses that benefits most from dermoscopic analysis as it usually displays a repetitive pattern, ie, uniformly distributed dotted vessels (histologically corresponding to dilated capillaries in regularly elongated dermal papillae) over a light or dull red background along with diffuse white scales (histologically corresponding to parakeratosis) (Figure 1A) [1]. Notably, psoriatic vessels are typically uniform in size, shape, and distance among each other [1,2]. In hyperkeratotic lesions, vascular structures may be poorly vis- ible and scale removal or the use of a fluid interface may facilitate their visualization [1,2]. In addition, scale removal may also display tiny red blood drops, the so-called dermoscopic "Auspitz sign" $[1,2]$.

Of note, vessels may sometimes appear as red globules (defined as round vessels having a diameter more than $0.1 \mathrm{~mm}$ ) on dermoscopic examination, especially on the legs (due to the higher hydrostatic pressure of this area) $[1,2,4]$. Both dotted and globular vessels look like dilated, elongated, and convoluted (bushy) capillaries under higher magnifications $(\times 100-\times 400)$ $[1,2,4]$. While differentiating dotted vessels from globular vessels has not been found to be of any help in the diagnostic accuracy in psoriasis, a recent study showed that the presence of globular vessels in psoriatic lesions is a negative response predictor to narrowband ultraviolet $\mathrm{B}$ (Nb-Uvb) phototherapy [4].

The association of uniform dotted vessels on a red background and diffuse white scales has been found to display good diagnostic accuracy for psoriasis, with a sensitivity of $84.9 \%$ and a specificity of $88.0 \%$ [5]. Indeed, although dotted/globular vessels and white scaling also characterize Bowen disease, in this condition vessels are more commonly distributed in a focal/clustered pattern and are different in size, shape, and distance among each other [6]. In addition, the presence of uniform dotted vessels vs short, fine telangiectasias is helpful in differentiating psoriatic patches from superficial basal cell carcinoma [7]. In this regard, a recent study introduced a dermoscopic diagnostic model for the differentiation of solitary psoriatic plaques from intraepidermal carcinoma and superficial basal cell carcinoma, stating that the concomitant presence of red dots, a homogeneous vascular pattern, and a light red background yields a diagnostic probability of $99 \%$ for psoriasis [7].

Importantly, uniform dotted/globular vessels may also be seen in lichen 
simplex chronicus and secondary lichenification [2]. Unlike psoriasis, however, in these conditions vessels are typically surrounded by a white halo due to the presence of hypergranulosis [2].

The dermoscopic features seen in specific subtypes of psoriasis do not differ significantly $[1,8]$. In fact, the dermoscopic pattern of the disease in specific body sites is identical to that of plaque psoriasis, with variations in the amount of scaling depending on the localization of the lesions [8]. In psoriatic balanitis and inverse psoriasis, lesions lack scaling, but the typical vascular pattern of regularly distributed red dots is evident on dermoscopic examination $[6,8]$. Little or absent scaling is also typical of guttate psoriasis as this form of psoriasis is eruptive, thereby having little hyperkeratosis [9]. Conversely, in scalp or palmoplantar psoriasis, the thick hyperkeratotic surface of the plaques does not allow the visualization of the underlying vascular structures, which are highlighted after removal of the scales $[8,10,11]$. In pustular psoriasis, palmoplantar and generalized forms, yellow globules (pustules) and crusts are also visible along with dotted vessels and white scaling, while follicular psoriasis is characterized by white follicular keratotic plugs surrounded by uniform dotted vessels [1,2].

The "red globular ring" pattern, with vessels distributed in a networklike arrangement, is another less common (but specific) vascular pattern visible in plaque-type psoriasis lesions $[1,2]$.

As mentioned above, dermoscopy may also be helpful in treatment evaluation and monitoring. Apart from the negative predictive value of globular vessels when psoriasis is treated with Nb-Uvb phototherapy, it has been demonstrated that hemorrhagic dots are a positive predictive sign suggesting a favorable clinical outcome in psoriasis treated with biological agents [12]. Finally, dermoscopy can reveal an otherwise not clinically evident skin atrophy following treatment with potent topical steroids by displaying telangiectatic vessels $[1,2]$.

\section{Eczematous Dermatitis}

Eczematous dermatitis consists of several distinct clinical entities that share the presence of spongiosis on histology, eg, atopic dermatitis, allergic contact dermatitis, stasis dermatitis, and asteatotic eczema [1,2].

The dermoscopic hallmarks of eczematous dermatitis include dotted vessels distributed in clusters or randomly (unspecific arrangement), corresponding to dilated capillaries in irregularly elongated dermal papillae, and yellow scales and serocrusts, resulting from hyperkeratosis and spongiosis/ exocytosis (Figure 1B) [1,2]. Hemorrhages may also be seen as a result of intense itching [1,2].

Of note, the dermoscopic pattern of eczematous dermatitis varies according to the disease stage [1,2]. In detail, in acute phases yellow serocrusts and dotted vessels distributed in clusters or randomly are typically seen, while more or less uniform dotted vessels surrounded by a white halo are the main dermoscopic features in chronic phases (lichenification) [1,2]. Obviously, overlapping pictures are often seen in clinical practice [1,2].

Importantly, some subtypes of eczematous dermatitis may display peculiar additional features. In particular, stasis dermatitis often displays globular or glomerular vessels due to the presence of a higher hydrostatic pressure, chronic hand eczema commonly shows brownish-salmon-colored dots/ globules (representing spongiotic vesicles), and asteatotic eczema frequently reveals white scales having a double free edge ("rail-like" appearance) [1,2,10].

A case-control study has demonstrated that dermoscopy may support the differential diagnosis between chronic, resistant-to-treatment or relapsing eczema and patch-stage mycosis fungoides [13]. In detail, patches of mycosis fungoides show thin linear vessels alone or in combination with red dots, forming the so-called "spermatozoon-like" structures, while dermatitis typically lacks linear vessels, unless overtreated with topical steroids [13].

\section{Lichen Planus}

The dermoscopic hallmark of lichen planus is represented by Wickham striae (Figure 1C), which histologically correspond to hypergranulosis [1,2]. In addition to their network-like appearance, Wickham striae may less commonly display other morphologies on dermoscopy, including linear, "radial streaming," annular, round, "leaf venation" (delicate secondary striae branching from the centered whitish venation, linked together at either end, mimicking the crystal structure of snow) and "starry sky" (clustered, follicular white dots) aspect [1,2]. Notably, Wickham striae are typically white, yet they may also appear yellow or blue, respectively, on palmoplantar areas and in darkskinned patients [1,2].

Importantly, network-like white structures similar to Wickham striae may also be seen in scarring/resolving lesions of several dermatoses (pseudoWickham striae), eg, discoid lupus erythematosus, nodular scabies, and prurigo nodularis $[1,2]$. They are due to dermal fibrosis and may be distinguished from Wickham striae on the basis of the vascular pattern as they usually show vessels that are significantly more dilated than those visible in Wickham striae [1,2].

Additional findings visible in active lesions include the following: (1) dotted, globular, and/or linear vessels, mainly detectable at the periphery of the lesion and less commonly showing a perifollicular or diffuse arrangement; (2) white/ yellow dots; and (3) pigmented structures (dots, globules, and/or reticular or cloud-like areas) [1,2].

Although all the above-mentioned findings may sometimes coexist in a 
single lesion, dermoscopic patterns of lichen planus usually vary according to disease stage. [1,2]. Indeed, while early papules usually show subtle Wickham striae over a reddish background, mature lesions display well-represented Wickham striae and peripheral vessels. $[1,2]$. Both of such structures tend to fade over time, concomitantly to the gradual appearance of pigmented structures (Figure 1C) [1,2]. In long-standing lesions, pigmentary findings are often the only visible clue [1].

Some clinical variants of lichen planus may reveal peculiar features [1,14]. Relevant examples include annular lichen planus, in which Wickham striae appear as a peripheral annular white structure with capillaries or pigmentary findings (according to lesion duration), and hypertrophic lichen planus, typically displaying follicular keratotic plugs $[1,14]$. In this last variant, Wickham striae are often not visible because they are covered by the overlying hyperkeratosis [1].

Apart from diagnostic purposes, dermoscopic examination may also be used to assess the likelihood of postinflammatory pigmentation persistence, with homogeneous, structureless, and light brown areas devoid of granularity being correlated with a shorter duration and granular pigmentation being associated with a longer course, and to monitor the evolution of lesions after therapy [15].

\section{Lichen Nitidus}

Dermoscopic examination of lichen nitidus typically reveals roundish, welldefined, white areas devoid of physiological skin markings (Figure 1D) [16]. The absence of skin markings is a very relevant dermoscopic clue to recognize such a dermatosis as it is related to a quite characteristic histological finding, ie, flattening of the epidermis overlying the inflammatory infiltrate [16]. However, lesions on the penis may retain skin markings, probably because they
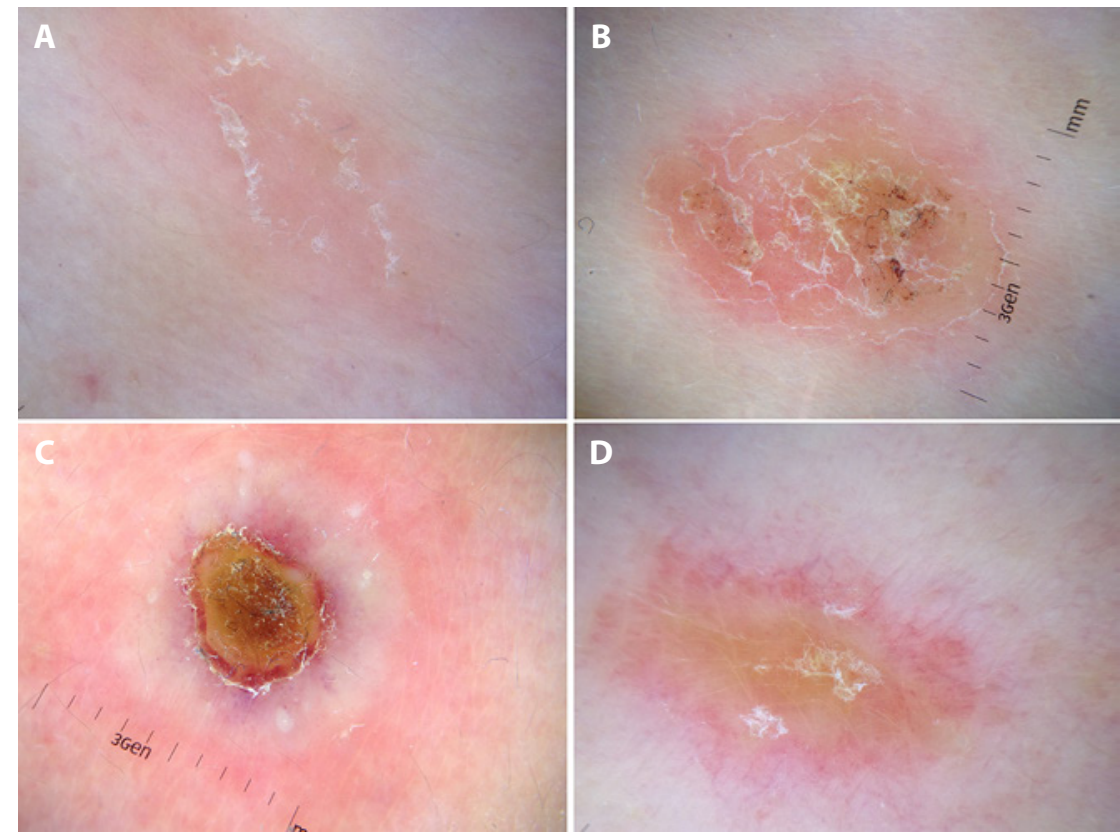

D

Figure 2. Dermoscopy of pityriasis rosea shows the characteristic peripheral whitish scaling (“collarette" sign). No vessels are seen (A); pityriasis rosea in an atopic patient displays yellow serocrusts/scaling along with the peripheral whitish scaling collarette (B). Central amorphous brownish crust surrounded by a peripheral scaling collarette and a purpuric halo is visible in a case of pityriasis lichenoides et varioliformis acuta $(\mathrm{C})$. Dermoscopic examination of pityriasis lichenoides chronica reveals an orange structureless area along with nondotted vessels (linear-irregular and branching vessels), white scaling, and sparse dotted vessels (D). [Copyright: @2019 Errichetti.]

are more pronounced or deep in this district [16].

Dermoscopic assessment may be helpful in differentiating lichen nitidus from its main differential diagnoses, including follicular eczema and frictional lichenoid dermatosis, respectively characterized by roundish, equidistant, keratotic, whitish areas with blurry margins and discrete, more or less defined, whitish areas with retention of the normal skin furrows and regularly arranged dotted vessels [16].

\section{Pityriasis Rosea}

Both the herald patch and secondary lesions of pityriasis rosea typically show a characteristic peripheral whitish scaling ("collarette" sign) as well as dotted vessels, which, unlike psoriasis, are distributed in an irregular or focal pattern (Figure 2A); diffuse or localized yellowish orange structureless areas may be visible as well $[1,2,5]$. Interestingly, an eczematous reaction may occur on the background of pityriasis rosea, especially in atopic patients, with yellow serocrusts/scaling and clustered dotted vessels visible on dermoscopy along with the peripheral collarette scaling (Figure 2B) [1,2,5].

\section{Pityriasis Lichenoides}

The term pityriasis lichenoides encompasses a spectrum of diseases that includes 2 main variants, ie, pityriasis lichenoides et varioliformis acuta (PLEVA) and pityriasis lichenoides chronica, although intermediate or overlapping forms do exist $[17,18]$.

Dermoscopic features of PLEVA and pityriasis lichenoides chronica differ significantly. Specifically, dermoscopic appearance of PLEVA varies according to the "lesion age," with very early lesions commonly displaying a purpuric 
aspect (more or less diffuse hemorrhagic areas due to erythrocyte extravasation), mature lesions usually showing a central amorphous brownish crust (due to epidermal necrosis) (Figure 2C), and healing lesions often featuring a central white area (due to fibrosis) [1,2,17]. A rim of pinpoint and/or linear vessels with a targetoid aspect may be seen at the periphery of the lesions [1,2,17]. These vascular structures appear as dilated and convoluted vessels at higher magnification, with some of them showing a glomerular pattern or linear aspect $[1,2,17]$. In addition, a peripheral scaling collarette having an inner free edge is often evident in all the stages, especially in mature (Figure 2C) and healing lesions [1,2,17].

On the other hand, pityriasis lichenoides chronica typically displays orangeyellowish

structureless areas (corresponding to hemosiderin deposits in the dermis due to erythrocyte extravasation) and nondotted vessels (including globular, linear-irregular, and/or branching vessels) (Figure 2D); diffuse and/or peripheral whitish scaling, focally distributed dotted vessels, hemorrhagic spots, and hypopigmented areas are additional dermoscopic findings [9]. Of note, the presence of hypopigmented areas is more common in long-standing lesions, which often display focal postinflammatory hypopigmentation. Importantly, in dark-skinned patients, orangish areas are difficult to see [9].

\section{Pityriasis Rubra Pilaris}

Dermoscopy has been found to be helpful in supporting the diagnosis of several clinical manifestations of both classic pityriasis rubra pilaris (PRP) and circumscribed juvenile PRP.

In detail, papular lesions of classic PRP usually reveal round/oval yellowish areas surrounded by vessels of mixed morphology (ie, linear and dotted) (Figure $3 \mathrm{~A}$ ) and often centered by central keratin plugs, whereas palmoplantar
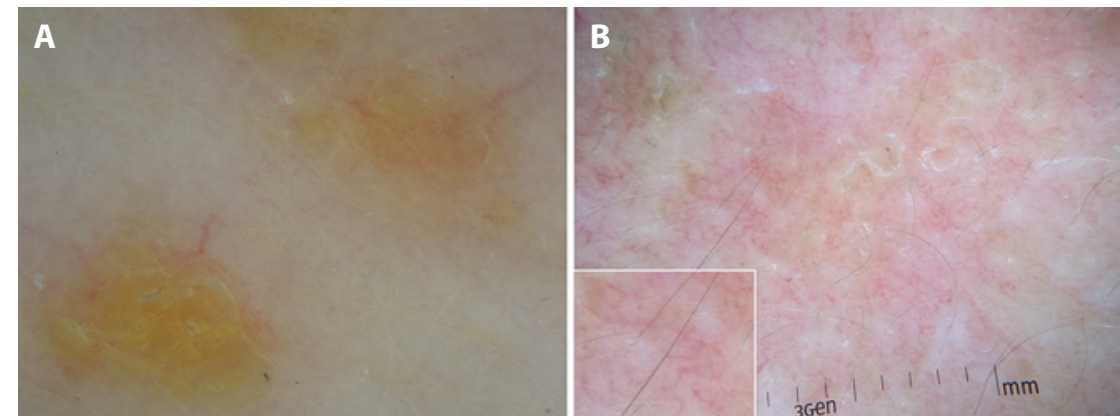

Figure 3. Pityriasis rubra pilaris features a roundish, yellowish area surrounded by vessels of mixed morphology (ie, linear and dotted) (A). Dermoscopy in subacute lupus erythematosus reveals white scales and a mixed vascular pattern (ie, dotted and linear vessels in this case; visualized more clearly in the box) over a pinkish-reddish background (B). [Copyright: C)2019 Errichetti.]

keratoderma is typified by orange structureless areas $[1,2,11]$. In erythrodermic stage, PRP features orange blotches and islands of nonerythematous (spared) skin displaying reticular vessels; unspecific whitish scaling and scattered dotted vessels over a reddish background may also be seen [18].

Dermoscopic assessment of lesions on elbows and knees in circumscribed juvenile PRP often displays whitish keratotic follicular plugs with a yellow peripheral keratotic ring, surrounded by an erythematous halo with linear and/ or dotted vessels; whitish scaling is also visible [2]. Palmoplantar keratoderma in this form of PRP shows the same aspect as the classic variants [11].

\section{Subacute Lupus Erythematosus}

Subacute cutaneous lupus erythematosus is characterized by 2 constant dermoscopic findings, namely whitish scales (diffusely or peripherally distributed) and a mixed vascular pattern (at least 2 types of vessels among dotted, linear-irregular, linear and branching vessels) over a pinkish-reddish background (Figure 3B) [19]. Focally distributed orange-yellowish structureless areas due to dermal hemosiderin deposits may also be seen less commonly [19]. Unlike discoid lupus erythematosus, follicular plugs are typically not seen, and this finding may be helpful to dis- tinguish extrafacial discoid lupus erythematosus from subacute lupus erythematosus [1,2,19].

\section{Papulokeratotic Dermatoses}

\section{Porokeratosis}

The dermoscopic hallmark of all variants of porokeratosis is the presence of a well-defined, annular, peripheral, white hyperkeratotic structure ("white track") having 2 free edges which appears as similar to the outlines of a volcanic crater as observed from a high point (Figure 4A) [1]. Such a keratotic rim may be hyperpigmented in disseminated superficial actinic porokeratosis and in dark-skinned patients [1]. From a histological point of view, it corresponds to the "cornoid lamella." In this regard, dermoscopy may be helpful even in treatment monitoring [1].

The center of the lesions is usually whitish or brownish and may exhibit circular and/or linear whitish and/or hyperpigmented tracks, blue-gray dots, and dotted, linear, or globular vessels [1].

\section{Darier Disease and Grover Disease}

Because they have a strict histological similarity, Darier disease and Darier-like Grover disease share a similar dermo- 
scopic pattern [20-22]. Indeed, both are typically characterized by a central yellowish-brownish area having a starlike, branched polygonal or round-oval shape, resulting from compact hyperkeratosis and exocytosis (due to acantholysis), and a peripheral white halo corresponding to acanthosis (Figure 4B) [20-22].

The same dermoscopic pattern is visible in BRAF-inhibitor-induced acantholytic dyskeratosis owing to their similar histological background [1]. On the other hand, spongiotic histological subtype of Grover disease displays whitish scaling over a reddish-yellowish background [22].

Dotted and/or linear/irregular vessels are additional findings visible in both Darier disease and Darier-like and spongiotic Grover disease [20-22].

\section{Prurigo Nodularis}

The dermoscopic pattern of prurigo nodularis (both hyperkeratotic and excoriated lesions) consists of radially arranged whitish lines or peripheral whitish halo with some centrifugal coarse projections on a brownish and/ or reddish background, the so-called “white starburst pattern" (Figure 4C) [23]. In the center of the lesions, brownreddish or brown-yellowish crusts (Figure 4C), erosions, and/or hyperkeratosis or scales may also be seen [23].

Such a pattern is quite different from that of its main differential diagnoses, including hypertrophic lichen planus (see above), acquired reactive perforating collagenosis (ARPC), and nodular scabies [1,23,24]. Central round brownish-greenish/yellowish brown structureless area surrounded by a white keratotic collarette and an erythematous halo with or without dotted vessels ("trizonal concentric" pattern) is typically seen in ARPC (Figure 4D), while nodular scabies usually features dotted vessels, sometimes associated with the presence of mites ("hang glider sign") and/or burrows ("jet with condensation trails") [1,23,24].
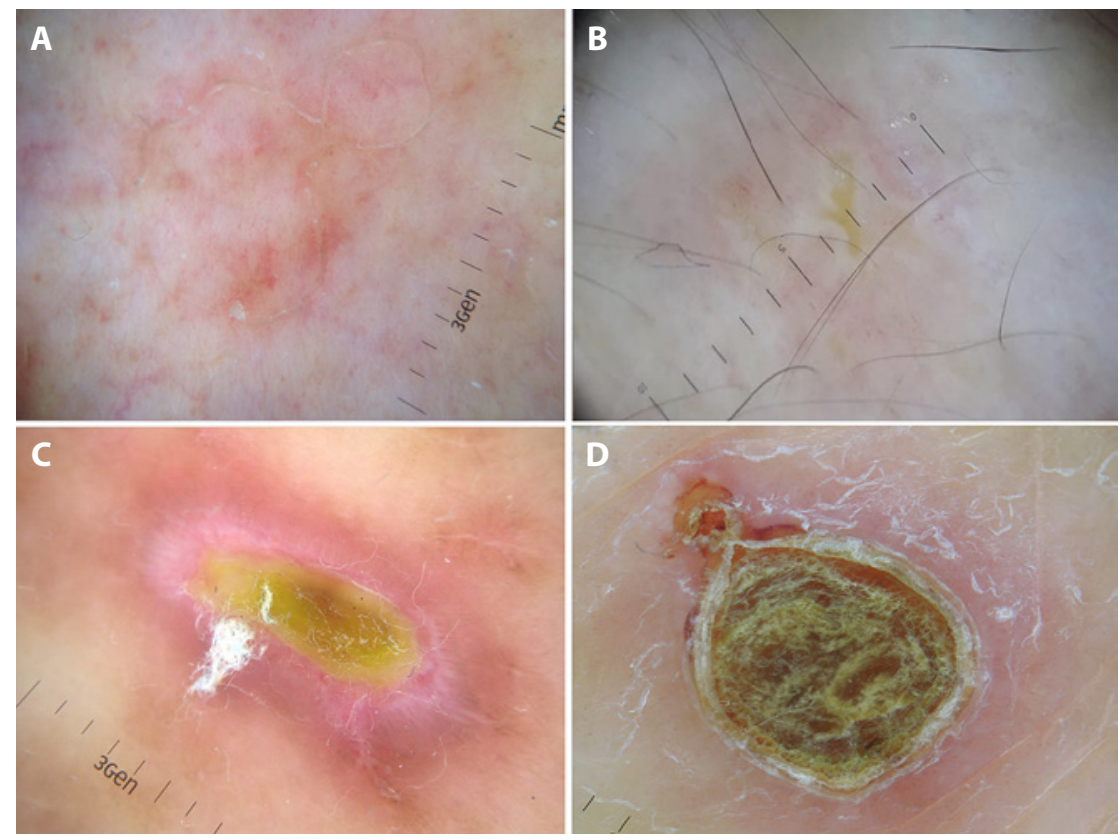

Figure 4. The typical white keratotic track having 2 free edges is visible in a case of porokeratosis (A). Darier disease characteristically reveals a central star-like, yellowish area surrounded by a peripheral white halo (B). The "white starburst" pattern (peripheral radial white striae over a reddish-brownish background) is visible in a case of prurigo nodularis; a central yellow crust is also present $(\mathrm{C})$. Acquired reactive perforating collagenosis displays a central round brownish-greenish structureless area surrounded by a white keratotic collarette and an erythematous halo ("trizonal concentric" pattern) (D). [Copyright: (C2019 Errichetti.]

\section{Erythematous Facial Dermatoses}

\section{Rosacea}

Erythemato-telangiectatic subtype is the most studied variant of rosacea $[25,26]$. It typically features a quite constant and specific dermoscopic pattern, namely linear vessels characteristically arranged in polygonal networks (vascular polygons) (Figure 5A) [25,26]. Additional dermoscopic findings may be seen but are poorly specific, including rosettes, follicular plugs, white-yellowish scales, pigmentation structures, and dilated follicles [25,26]. Pustules and orange structureless areas, often associated with vascular polygons, are typically seen in papulopustular and granulomatous rosacea, respectively $[1,2,25,26]$.

\section{Seborrheic Dermatitis}

Although a study on facial inflammatory dermatoses concluded that the most typical dermoscopic findings of seborrheic dermatitis consist of dotted vessels in a patchy distribution and fine yellowish scales (in combination or not with white scales) [25], it is not uncommon to observe facial lesions displaying a different vascular pattern, ie, linear branching vessels (with or without dotted vessels) [1,2]. Such a vascular pattern is even more common in scalp seborrheic dermatitis $[1,2]$. Additional, unspecific features of facial seborrheic dermatitis include follicular plugs, orange-yellowish areas, and whitish structureless areas [25].

\section{Demodicosis}

Demodicosis is an underrecognized facial dermatosis whose clinical presentation may mimic several dermatoses, mainly including rosacea and seborrheic dermatitis [27].

The dermoscopic hallmark of all clinical subtypes are the so-called 
“Demodex tails," which are white-yellowish, protruding, follicular keratotic plugs due to the presence of a mixture of keratotic material and mites in the follicles (Figure 5B) [27]. "Demodex follicular openings," which consist of round and coarse follicular openings containing white/yellow plugs surrounded by an erythematous halo, are also referred as typical of demodicosis and may represent nonprotruding Demodex tails [27].

Other unspecific dermoscopic findings may be observed (ie, diffuse erythema, scaling, pustules, and reticular dilated vessels) and their prevalence varies according to the subtypes of demodicosis [27].

\section{Discoid Lupus Erythematosus}

Dermoscopy of facial (and extrascalp in general) discoid lupus erythematosus reveals different features according to the disease stage $[1,25,28]$.

Early lesions are typified by white scales and follicular findings, namely follicular red dots surrounded by whitish halos ("inverse strawberry" pattern) or follicular whitish-yellowish keratotic plugs (visible as white rosettes on polarized-light dermoscopy) over a more or less erythematous background ("strawberry" pattern) (Figure 5C) [1,25,28]. Vessels of variable morphology (dotted, linear-irregular, and/or branching) may also be seen, especially at the periphery of the lesions [1,25,28].

On the other hand, late lesions display white structureless areas, pigmentary structures, hair loss, and telangiectatic linear-irregular, branching vessels and/or dotted/glomerular vessels $[1,25,28]$. Importantly, intermediatestage lesions may reveal a mixture of all the aforementioned features [1,25,28]. Less common dermoscopic findings include diffuse hyperkeratosis (hypertrophic discoid lupus erythematosus), dilated follicles, and yellowish scales $[1,25,28]$.
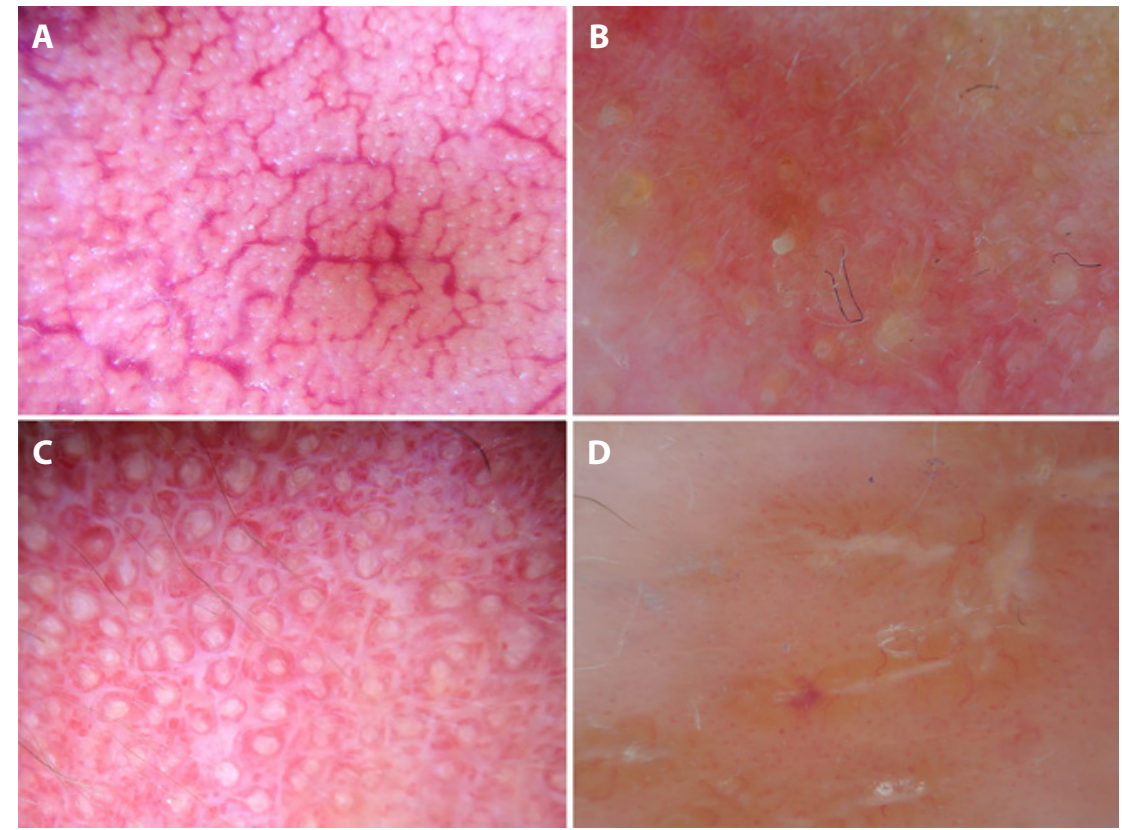

Figure 5. Erythemato-telangiectatic rosacea with its typical linear vessels arranged in polygonal networks (vascular polygons) (A). The so-called Demodex tails (white-yellowish, protruding, follicular keratotic plugs) are visible in demodicosis (B). White keratotic plugs over a reddish background are the typical dermoscopic features of active discoid lupus erythematosus (C). Dermoscopy of sarcoidosis reveals orange structureless areas with overlying focused linear vessels; white areas are also visible (D). [Copyright: C2019 Errichetti.]

\section{Granuloma Faciale}

Despite its name, granuloma faciale is a chronic leukocytoclastic vasculitis and not a granulomatous disease [1,25]. The presence of dilated follicular openings has been reported as the most characterizing dermoscopic feature of this condition [1,25]. Linear and/or branching dilated vessels are also commonly seen $[1,25]$. In addition, purpuric spots and orange structureless areas, histologically corresponding to erythrocyte extravasation and hemosiderin dermal deposits, may also be observed [1,25,29]. Less common, unspecific findings include perifollicular whitish halo, pigmentation structures, follicular plugs, yellowish scales, whitish streaks, and whitishgrayish structureless areas [1,25].

\section{Granulomatous Facial Diseases}

The main granulomatous dermatoses of the face include sarcoidosis, lupus vulgaris, and cutaneous leishmaniasis [30].
The first 2 conditions display an indistinguishable dermoscopic pattern, which is typified by focal or diffuse orange structureless areas and well-focused linear or branching vessels, which are usually located over the orange areas (Figure 5D) [30]. Other possible findings include milia-like cysts, erythema, whitish lines or structureless areas, follicular plugs, dilated follicles, pigmentation structures, and white and/or yellow scales [30].

On the other hand, even though leishmaniasis is a granulomatous dermatosis, orange structureless areas are visible only in a minority of patients (approximately $15 \%$ of cases) because they are covered by the frequent overlying epidermal changes (ie, ulcerations/ erosions, crusting, or hyperkeratosis/ scaling) [30-32]. According to the available literature, white/yellow follicular keratotic plugs (previously called "yellow tears") and white peripheral projections ("white starburst pattern") are the most characterizing features [30-32]. 

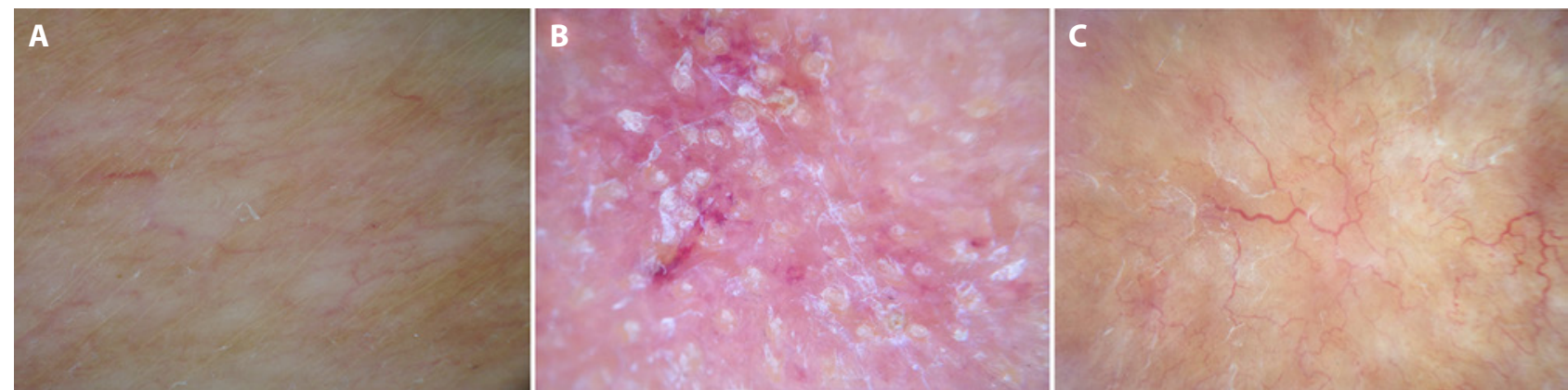

Figure 6. Morphea is characterized by dull white areas with blurry margins (“white clouds”) (A). Keratotic follicular plugs, white scales, and hemorrhagic spots over a white-pinkish background are visible in lichen sclerosus (B). Necrobiosis lipoidica: yellow-orange structureless areas and branching-serpentine vessels whose diameter decreases from the center to the periphery of the lesion (C). [Copyright: (ㅇ)2019 Errichetti.]

In addition, cutaneous leishmaniasis is often typified by vascular structures having a variable morphology, including hairpin, comma, glomerular, and/or corkscrew vessels [30-32]. Thrombotic vessels, yellowish hue, white scarring areas, milia-like cysts, pustules, and perilesional hypopigmented halo are further unspecific findings that may be seen [30-32].

\section{Sclero-atrophic Dermatoses}

\section{Morphea}

The most specific dermoscopic feature of morphea consists of white clouds, which are ill-defined dull white areas corresponding to deep dermal fibrosis (Figure 6A); erythematous areas, focused vessels (especially linear-irregular but also branching and dotted), crystalline structures, unfocused large purple vessels, yellowish areas, and pigmentary structures (structureless brown areas, reticular brown areas, and brown dots) are additional findings [33].

Of note, dermoscopic features may vary according to the disease stage, with erythematous areas and focused vessels (especially linear-irregular) being indicative of inflammatory phases and unfocused large purple vessels typical of atrophic stages [33]. However, the prevalence of white clouds does not differ significantly among inflammatory, sclerotic, and atrophic stages, thereby highlighting that lesions belonging to different clinical phases may display a dermoscopic (and histological) overlap [33].

\section{Lichen Sclerosus}

The hallmarks of cutaneous lichen sclerosus are represented by keratotic follicular plugs, corresponding to follicular hyperkeratosis, and well-defined, bright white patches, resulting from superficial fibrosis (Figure 6B) [33]. In addition, scaling and hemorrhagic spots have been shown to be quite specific for lichen sclerosus when compared to morphea [33]. Less common features include erythematous areas, focused vessels (especially linear-irregular and dotted), crystalline structures, unfocused large purple vessels, yellowish areas, and pigmentary structures (reticular brown areas and brown dots) [33].

Even in cutaneous lichen sclerosus there may be a variability of dermoscopic pattern according to the clinical disease stage, with erythematous areas and focused vessels being more common in inflammatory lesions and unfocused large purple vessels and yellowish areas being typical of atrophic stages, yet a dermoscopic overlap is possible as the prevalence of bright white areas does not differ significantly between inflammatory and sclerotic lesions [33]. Interestingly, follicular keratotic plugs have been found more commonly in clinically inflammatory- sclerotic and sclerotic lesions, thus underlining that such a dermoscopic clue is much more relevant and useful for active lesions than early inflammatory and late ones [33].

Mucosal (anogenital) lichen sclerosus reveals the same features as the cutaneous lesions (especially bright white areas and well-focused vessels), apart from the lack of follicular keratotic plugs [33].

\section{Necrobiosis Lipoidica}

Dermoscopy of necrobiosis lipoidica lesions typically shows yellow-orange structureless areas (Figure 6C), due to the presence of granulomatous inflammatory infiltrate and lipid deposits in the dermis, and well-focused vascular structures, whose morphology varies according to the disease stage, with dotted, globular, comma-shaped, and glomerular vessels more commonly seen in early stages or active lesional border, network-shaped, linear, and hairpin-like vessels more frequent in more developed (mature) lesions and branching-serpentine vessels being typical of advanced lesions [30]. Of note, diameter of branching-serpentine vessels typically decreases from the center to the periphery of the lesion due to the more marked epidermal atrophy in central areas (Figure 6C) [30].

Additional less common and poorly specific dermoscopic findings include ulcerations, whitish-yellowish crusts, 
whitish scaling, brownish reticular structures, and whitish structureless areas, with this last feature being more common in long-standing lesions, which are characterized by pronounced dermal fibrosis [30].

\section{Miscellaneous}

\section{Common Urticaria vs Urticarial Vasculitis}

Dermoscopy of common urticaria and urticarial vasculitis frequently shows a homogeneous erythematous background (avascular areas), yet such a feature is less common in late lesions of urticaria vasculitis [2]. Reticular/ linear vessels may be present in both diseases, but they are significantly more frequent in common urticaria [2]. Conversely, purple-red dots/globules, often on an orange-brown background, are highly indicative of urticarial vasculitis, as they are very rare in common urticaria [2].

\section{Capillaritis (Pigmented Purpuric Dermatoses) vs Vasculitis}

The term capillaritis refers to a group of chronic, benign, cutaneous diseases that are characterized by extravasation of erythrocytes in the skin with consequent hemosiderin deposition, including Schamberg disease, Doucas-Kapetanakis disease, Majocchi disease, Gougerot-Blum syndrome, and lichen aureus $[34,35]$. All of them are typified by the presence of focused reddish purpuric dots and/or globules over a brownishcoppery background (Figure 7A); in addition, peripheral telangiectatic vessels and yellow scales/crusts are seen in Majocchi disease and Doucas-Kapetanakis disease, respectively [34,35].

Unlike capillaritis, dermoscopy of small-vessel skin vasculitis may reveal blue-gray patches and purpuric globules/dots that are usually violaceous and blurrier (Figure 7B) because extravasated erythrocytes are located deeper [36].
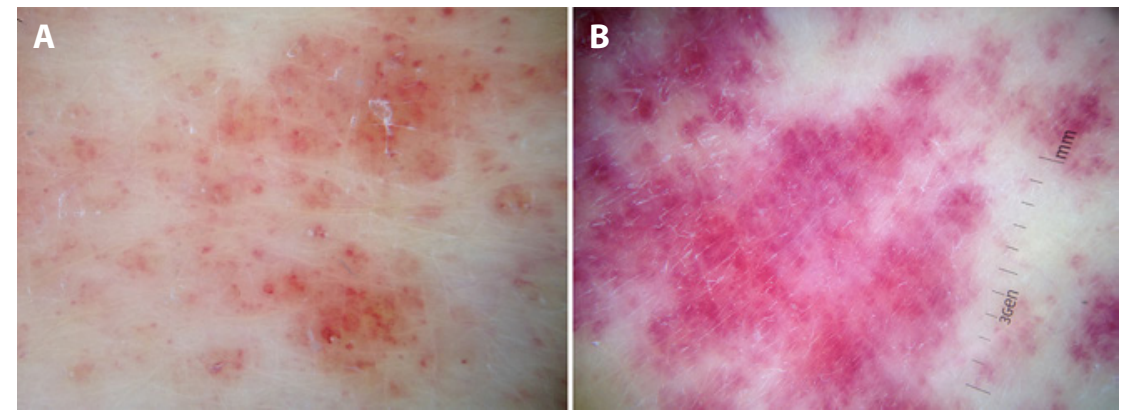

Figure 7. Schamberg disease: focused reddish purpuric dots and globules over a coppery background (A). Unlike capillaritis, dermoscopy of small-vessel skin vasculitis reveals blurrier violaceous purpuric globules (B). [Copyright: (C2019 Errichetti.]
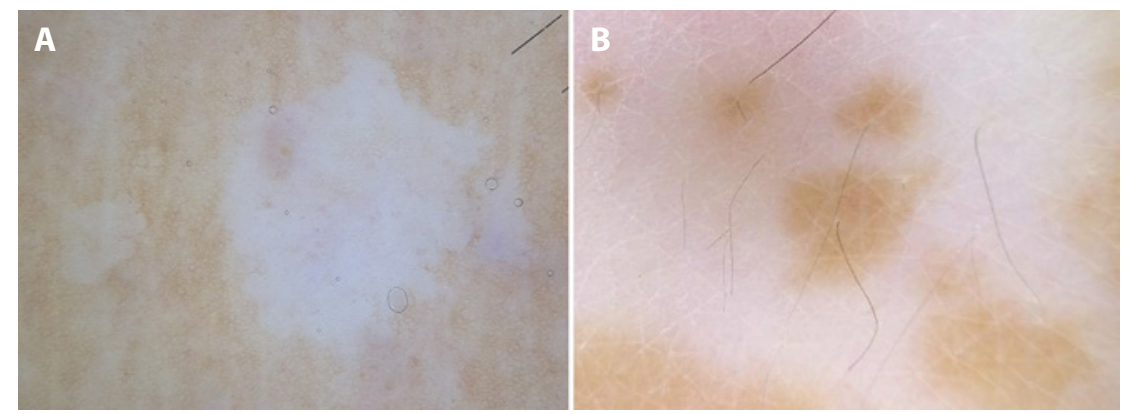

Figure 8. Idiopathic guttate hypomelanosis: well-defined roundish homogeneous whitish area ("cloudy" pattern) surrounded by patchy hyperpigmented network (A). Vitiligo: white areas and perifollicular pigmentation (B). [Copyright: (C2019 Errichetti.]

\section{Idiopathic Guttate Hypomelanosis vs Vitiligo}

Dermoscopy of idiopathic guttate hypomelanosis displays 2 main aspects, ie, the "cloudy sky-like" pattern (multiple small areas coalescing into irregular/polycyclic macules, with several white shades and both well- and illdefined edges) and the "cloudy" pattern (well- or ill-defined roundish homogeneous whitish areas) (Figure 8A), respectively more common in bigger and smaller lesions [37]. Notably, in both cases, patchy hyperpigmented network typically surrounds white areas (Figure 8A) [37].

On the other hand, well-demarcated, dense/glowing, white areas are the most common dermoscopic findings in vitiligo (Figure 8B) [1]. Although not present in all lesions, white hairs and perifollicular pigmentation (Figure 8B) are considered the most specific features of vitiligo, with the latter finding more frequently seen in repigmenting or progressing lesions than stable lesions [1]. Other possible features include intralesional pigmentary islands, perilesional hyperpigmentation, reversed pigmentary network, reticular pigmentation, and telangiectasias [1].

\section{Lichen Pigmentosus vs Ashy Dermatosis}

Both lichen pigmentosus and ashy dermatosis are characterized by pigmented dots ("peppering") [38]. However, in the former dermatosis, dots are usually brownish and larger (Figure 9A) than those seen in the latter condition, which typically displays smaller and graybluish dots over a bluish background (Figure 9B) [38]. Such differences are the result of the different level of melanophages/melanin deposits (superficial dermis in lichen pigmentosus and deep papillary and reticular dermis in ashy dermatosis) [38]. 


\section{Pityriasis Versicolor vs Gougerot- Carteaud Syndrome}

Distinguishing such conditions may be sometimes difficult on clinical grounds, and dermoscopy may provide some clues to facilitate their differential diagnosis [1,39]. In particular, pityriasis versicolor is characterized by fine whitish scales localized in the skin furrows and a brownish background, while Gougerot-Carteaud syndrome typically shows fine whitish scaling associated with brownish, homogeneous, more or less defined, polygonal, flat globules separated by whitish/pale striae creating a cobblestone appearance or brownish areas presenting a "sulci and gyri” pattern [1,39].

\section{Balanitis}

According to a recent study, dermoscopic examination may provide useful information on common forms of balanitis [6]. In particular, psoriatic balanitis and Zoon balanitis are the most recognizable forms, with the former being characterized by homogeneous dotted vessels distributed in a uniform pattern and the latter typically displaying focal or diffuse orange areas along with focused linear curved vessels [6]. On the other hand, cottage cheese-like structures (representing sparse white coating corresponding to Candida yeast colony growth) showed a strong correlation with candidal balanitis [6]. All the above-mentioned forms of balanitis may be distinguished from erythroplasia of Queyrat, which features glomerular vessels distributed in a focal or diffuse pattern [6]. Notably, differentiating dotted from globular vessels on hand-held dermoscopic examination may be challenging [6]. However, in erythroplasia of Queyrat, vessels are typically more heterogeneous in shape, size, and distance among each other [6].

\section{Mastocytoses}

Dermoscopic features of cutaneous mastocytoses vary according to the disease
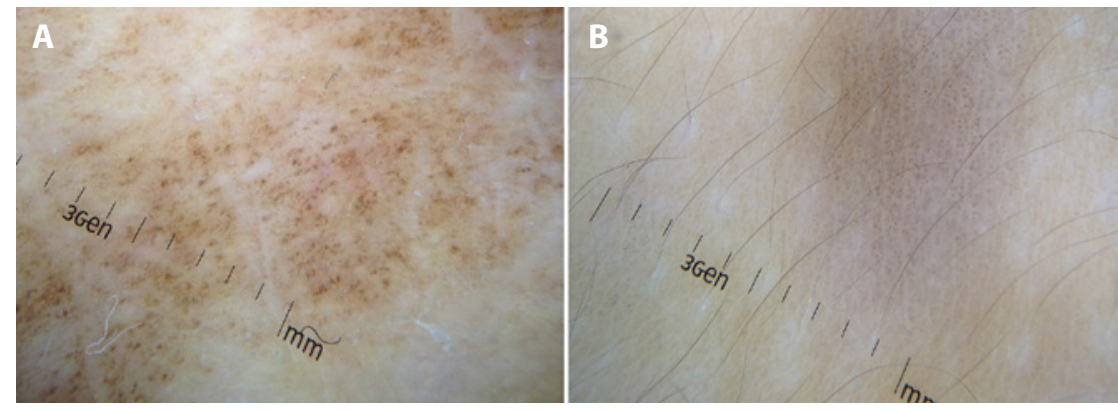

Figure 9. Brownish dots in lichen pigmentosus (A). In ashy dermatosis dots are smaller and bluish (B). [Copyright: (O2019 Errichetti.]
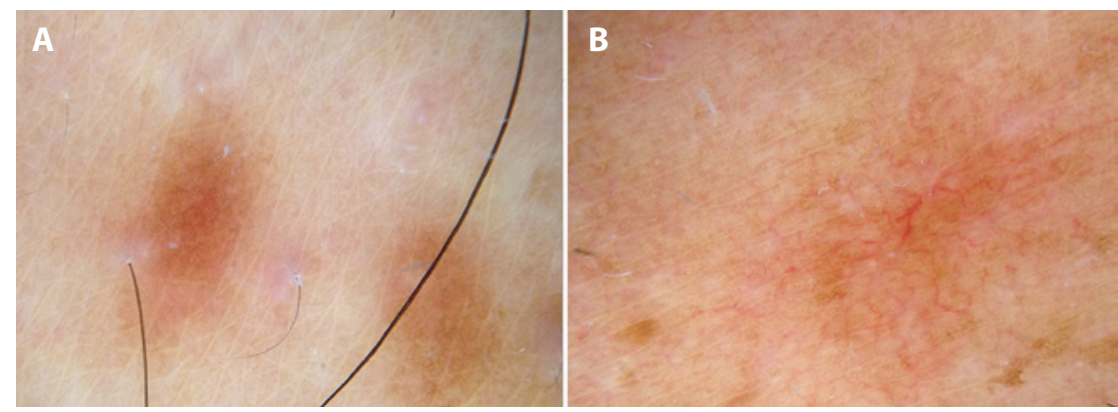

Figure 10. Coarse brownish network in urticaria pigmentosa (A). Telangiectasia macularis eruptiva perstans reveals tortuous linear vessels on a brownish base (B). [Copyright: (0)2019 Errichetti.]

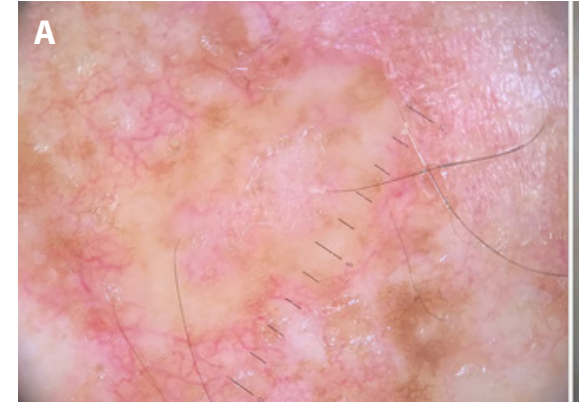

B

Figure 11. In both "palisading granuloma" and "interstitial" histological variants of granuloma annulare, dermoscopy shows unfocused vessels having a variable morphology (dotted, linear-irregular, and/or branching) over a more or less evident pinkish-reddish background $(\mathrm{A}, \mathrm{B})$. However, the former variant also features yellowish orange areas (A). [Copyright: (C)2019 Errichetti.]

subtype [1]. In detail, urticaria pigmentosa typically reveals either a homogeneous light-brown background or a coarse pigment network (Figure 10A), while telangiectasia macularis eruptiva perstans (TMEP) is mainly characterized by reticular or tortuous linear vessels on an erythematous/brownish base (Figure 10B) [1]. A brownish network may sometimes be appreciated in TMEP [1]. Finally, diffuse/multifocal yellow-orange discoloration, diffuse light-brown discoloration, and brownish network-like appearance may be seen in mastocytoma, with the last 2 patterns more commonly visible in regressing phases $[1,40]$.

\section{Granuloma Annulare}

The main dermoscopic clue (prevalence rate of $88.0 \%$ ) of granuloma annulare is represented by unfocused vessels having a variable morphology (dotted, linear-irregular, and/or branching) over a more or less evident pinkish-reddish background (Figure 11A,B) [41]. Whitish (irregular or globular) and yellowish 
orange (focally or diffusely distributed) areas represent the most common nonvascular findings [41]. Of note, dermoscopic appearance of granuloma annulare significantly varies according to its histological subtype, with a strict association between yellowish orange structureless areas and palisading granuloma histological variant (Figure 11A) as they are usually absent in lesions having an interstitial histological pattern (Figure 11B) [41].

\section{References}

1. Errichetti E, Stinco G. Dermoscopy in general dermatology: a practical overview. Dermatol Ther (Heidelb). 2016;6(4):471-507.

2. Errichetti E, Stinco G. The practical usefulness of dermoscopy in general dermatology. G Ital Dermatol Venereol. 2015;150(5):533-546.

3. Errichetti E, Zalaudek I, Kittler H, et al. Standardization of dermoscopic terminology and basic dermoscopic parameters to evaluate in general dermatology (non-neoplastic dermatoses): an expert consensus on behalf of the International Dermoscopy Society. Br J Dermatol. 2019 May 11. Epub ahead of print. doi: 10.1111/bjd.18125.

4. Errichetti E, Stinco G. Clinical and dermoscopic response predictors in psoriatic patients undergoing narrowband ultraviolet B phototherapy: results from a prospective study. Int J Dermatol. 2018;57(6):681-686.

5. Lallas A, Kyrgidis A, Tzellos TG, et al. Accuracy of dermoscopic criteria for the diagnosis of psoriasis, dermatitis, lichen planus and pityriasis rosea. $\mathrm{Br}$ J Dermatol. 2012;166(6):1198-1205.

6. Errichetti E, Lallas A, Di Stefani A, et al. Accuracy of dermoscopy in distinguishing erythroplasia of Queyrat from common forms of chronic balanitis: results from a multicentric observational study. J Eur Acad Dermatol Venereol. 2019;33(5):966-972.

7. Pan Y, Chamberlain AJ, Bailey M, Chong AH, Haskett M, Kelly JW. Dermatoscopy aids in the diagnosis of the solitary red scaly patch or plaque-features distinguishing superficial basal cell carcinoma, intraepidermal carcinoma, and psoriasis. J Am Acad Dermatol. 2008;59(2):268274.
8. Lallas A, Apalla Z, Argenziano G, et al. Dermoscopic pattern of psoriatic lesions on specific body sites. Dermatology. 2014;228(3):250-254.

9. Errichetti E, Lacarrubba F, Micali G, Piccirillo A, Stinco G. Differentiation of pityriasis lichenoides chronica from guttate psoriasis by dermoscopy. Clin Exp Dermatol. 2015;40(7):804-806.

10. Errichetti E, Stinco G. Dermoscopy in differential diagnosis of palmar psoriasis and chronic hand eczema. J Dermatol. 2016;43(4):423-425.

11. Errichetti E, Stinco G. Dermoscopy as a supportive instrument in the differentiation of the main types of acquired keratoderma due to dermatological disorders. J Eur Acad Dermatol Venereol. 2016;30(12):e229-e231.

12. Lallas A, Argenziano G, Zalaudek I, et al. Dermoscopic hemorrhagic dots: an early predictor of response of psoriasis to biologic agents. Dermatol Pract Concept. 2016;6(4):7-12.

13. Lallas A, Apalla Z, Lefaki I, et al. Dermoscopy of early stage mycosis fungoides. J Eur Acad Dermatol Venereol. 2013;27(5):617-621.

14. Güngör Ş, Topal IO, Göncü EK. Dermoscopic patterns in active and regressive lichen planus and lichen planus variants: a morphological study. Dermatol Pract Concept. 2015;5(2):45-53.

15. Vázquez-López F, Maldonado-Seral C, López-Escobar M, Pérez-Oliva N. Dermoscopy of pigmented lichen planus lesions. Clin Exp Dermatol. 2003;28(5):554-555.

16. Errichetti E, Stinco G. Comment on "Dermatoscopic features of lichen nitidus." Pediatr Dermatol. 2018;35(6):879-880.

17. Lacarrubba F, Verzì AE, Dinotta F, Scavo S, Micali G. Dermatoscopy in inflammatory and infectious skin disorders. G Ital Dermatol Venereol. 2015;150(5):521531.

18. Errichetti E, Piccirillo A, Stinco G. Dermoscopy as an auxiliary tool in the differentiation of the main types of erythroderma due to dermatological disorders. Int J Dermatol. 2016;55(12):e616-e618.

19. Errichetti E, Piccirillo A, Viola L, Stinco G. Dermoscopy of subacute cutaneous lupus erythematosus. Int J Dermatol. 2016;55(11):e605-e607.

20. Errichetti E, Stinco G, Lacarrubba F, Micali G. Dermoscopy of Darier's disease. J Eur Acad Dermatol Venereol. 2016;30(8):1392-1394.
21. Errichetti E, Maione V, Pegolo E, Stinco G. Dermoscopy: a useful auxiliary tool in the diagnosis of type 1 segmental Darier's disease. Dermatol Pract Concept. 2016;6(2):53-55.

22. Errichetti E, De Francesco V, Pegolo E, Stinco G. Dermoscopy of Grover's disease: variability according to histological subtype.J Dermatol. 2016;43(8):937-939.

23. Errichetti E, Piccirillo A, Stinco G. Dermoscopy of prurigo nodularis. J Dermatol. 2015;42(6):632-634.

24. Suh KS, Han SH, Lee KH, et al. Mites and burrows are frequently found in nodular scabies by dermoscopy and histopathology. J Am Acad Dermatol. 2014;71(5):1022-1023.

25. Lallas A, Argenziano G, Apalla Z, et al. Dermoscopic patterns of common facial inflammatory skin diseases. J Eur Acad Dermatol Venereol. 2014;28(5):609-614.

26. Lallas A, Argenziano G, Longo C, et al. Polygonal vessels of rosacea are highlighted by dermoscopy. Int J Dermatol. 2014;53(5):e325-327.

27. Segal R, Mimouni D, Feuerman H, Pagovitz O, David M. Dermoscopy as a diagnostic tool in demodicidosis. Int $J$ Dermatol. 2010;49(9):1018-1023.

28. Lallas A, Apalla Z, Lefaki I, et al. Dermoscopy of discoid lupus erythematosus. Br J Dermatol. 2013;168(2):284-288.

29. Jardim MML, Uchiyama J, Kakizaki P, Valente NYS. Dermoscopy of granuloma faciale: a description of a new finding. $A n$ Bras Dermatol. 2018;93(4):587-589.

30. Errichetti E, Stinco G. Dermatoscopy of granulomatous disorders. Dermatol Clin. 2018;36(4):369-375.

31. Taheri AR, Pishgooei N, Maleki M, et al. Dermoscopic features of cutaneous leishmaniasis. Int J Dermatol. 2013;52(11):1361-1366.

32. Yücel A, Günaşti S, Denli Y, Uzun S. Cutaneous leishmaniasis: new dermoscopic findings. Int J Dermatol. 2013;52(7):831837.

33. Errichetti E, Lallas A, Apalla Z, Di Stefani A, Stinco G. Dermoscopy of morphea and cutaneous lichen sclerosus: clinicopathological correlation study and comparative analysis. Dermatology. 2017;233(6):462-470.

34. Zalaudek I, Ferrara G, Brongo S, et al. Atypical clinical presentation of pigmented purpuric dermatosis. J Dtsch Dermatol Ges. 2006;4(2):138-140.

35. Zaballos P, Puig S, Malvehy J. Dermoscopy of pigmented purpuric dermatoses (lichen aureus): a useful tool 
for clinical diagnosis. Arch Dermatol. 2004;140(10):1290-1291.

36. Choo JY, Bae JM, Lee JH, et al. Bluegray blotch: a helpful dermoscopic finding in optimal biopsy site selection for true vasculitis. J Am Acad Dermatol. 2016;75(4):836-838.

37. Errichetti E, Stinco G. Dermoscopy of idiopathic guttate hypomelanosis. J Dermatol. 2015;42(11):1118-1119.
38. Errichetti E, Angione V, Stinco G. Dermoscopy in assisting the recognition of ashy dermatosis. JAAD Case Rep. 2017;3(6):482-484.

39. Errichetti E, Maione V, Stinco G. Dermatoscopy of confluent and reticulated papillomatosis (Gougerot-Carteaud syndrome). J Dtsch Dermatol Ges. 2017;15(8):836-838.
40. Vano-Galvan S, Alvarez-Twose I, De las Heras E, et al. Dermoscopic features of skin lesions in patients with mastocytosis. Arch Dermatol. 2011;147(8):932-940.

41. Errichetti E, Lallas A, Apalla Z, Di Stefani A, Stinco G. Dermoscopy of granuloma annulare: a clinical and histological correlation study. Dermatology. 2017;233(1):74-79. 